\title{
Common ECG Lead Placement Errors. Part I: Limb lead Reversals
}

\author{
Allison V. Rosen, ${ }^{1}$ Sahil Koppikar, ${ }^{2}$ Catherine Shaw, ${ }^{3}$ Adrian Baranchuk. ${ }^{3}$
}

\begin{abstract}
Background: Electrocardiography (ECG) is a very useful diagnostic tool. However, errors in placement of ECG leads can create artifacts, mimic pathologies, and hinder proper ECG interpretation. It is important for members of the health care team to be able to recognize the common patterns resulting from lead placement errors. Methods: 12-lead ECGs were recorded in a single male healthy subject in his mid 20s. Six different limb lead reversals were compared to ECG recordings from correct lead placement. Results: Classic ECC patterns were observed when leads were reversed. Methods of discriminating these ECG patterns from true pathologic findings were described. Conclusion: Correct recording and interpretation of ECGS is key to providing optimal patient care. It is therefore crucial to be able to recognize common ECG patterns that are indicative of lead reversals.
\end{abstract}

Keywords: Cardiology, Electrocardiography, Medical Errors, Limb Lead Reversal, Medical Education (Source: MeSH-NLM).

About the Author: Allison Rosen is currently in her third year of medical school at Queen's University, Kingston, Canada. She is the Editor-in-Chief of the Queen's Medical Review and an active member of the Class Council.

\section{Introduction}

Electrocardiography (ECG) remains a very useful diagnostic tool in the 21st century.' In this clinical era, it remains one of the most useful diagnostic tools in medicine. ${ }^{2}$ It is both inexpensive and very efficacious in many different clinical scenarios. However, proper ECG recording is of utmost importance in order to ensure an accurate interpretation of findings. ${ }^{3}$ Due to the structure of different healthcare programs, ECGs may be recorded not by expert technicians, but by whoever is available..$^{4-6}$ This has led to multiple errors in interpreting results..$^{7-10}$ Erroneous interpretation of ECGs has led to improper treatment of patients. ${ }^{11,12}$ Prior work has identified some common ECG placement errors and their resulting artifacts. ${ }^{13}$ The REVERSE mnemonic is a useful tool that can be used to identify abnormal electrocardiographic findings and the common placement errors that can elicit such findings (Table 1 ).?
The purpose of this study is to review some of the most common recording errors. For the purpose of clarity, this work has been divided into two parts. Part 1 is dedicated to limb lead reversals. Part 2 will deal with precordial placement errors.

\section{Methods}

A 24-year-old healthy male on no medications, and with no medical conditions, served as a model for ECC recordings. Recordings were obtained with the help of an expert technician (CS). A 12-lead ECG (General Electric, Mac 5500, United States) set up at $150 \mathrm{~Hz}, 25.0 \mathrm{~mm} / \mathrm{s}$, and $10.0 \mathrm{~mm} / \mathrm{V}$ was taken in a normal supine position. Arm electrode placements were selected in accordance with the AHA recommendations (Figure 1). ${ }^{14}$ A series of lead reversals were conducted in order to recreate these classic patterns.

Table 1. REVERSE mnemonic: an easy approach to remember the most frequent causes of electrocardiographic placement errors and artifacts.?

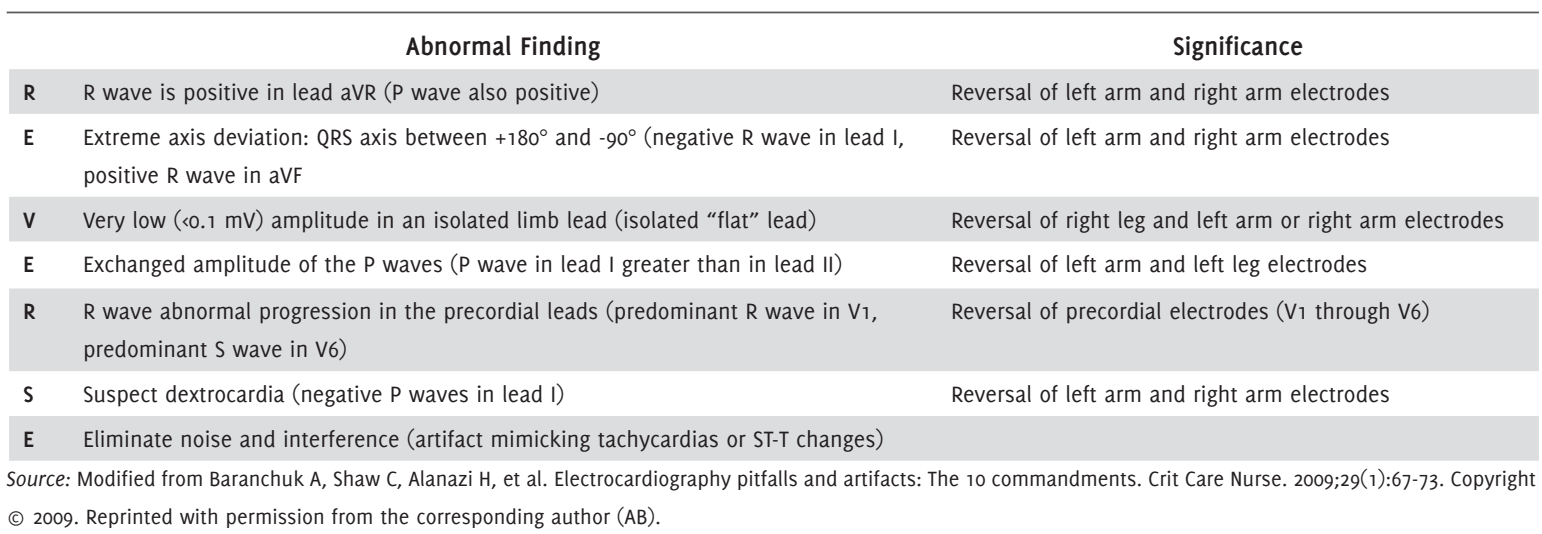

'Queen's University School of Medicine, Kingston, Ontario, Canada.

${ }^{2}$ Department of Internal Medicine, Kingston General Hospital, Queen's University, Kingston, Ontario, Canada.

${ }^{3}$ Heart Rhythm Service, Kingston General Hospital, Queen's University, Kingston, Ontario, Canada.

Correspondence:

Adrian Baranchuk, MD FACC FRCPC. Associate Professor of Medicine, Cardiac Electrophysiology and Pacing, Kingston General Hospital, Queen's University.

Address: Cardiac Electrophysiology and Pacing, Kingston General Hospital K7L 2V7, Queen's University, Kingston, Canada.

Email: barancha@kgh.kari.net 
Figure 1. 12 lead ECG depicting correct lead placement. (A) ECG recording. (B) Correct lead placements on chest and arms.

A

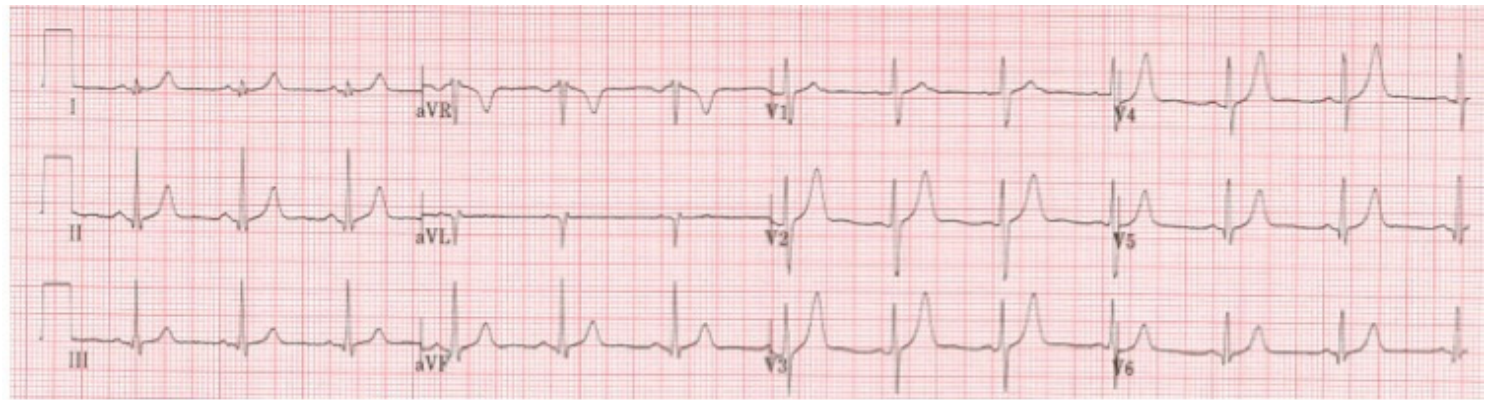

B

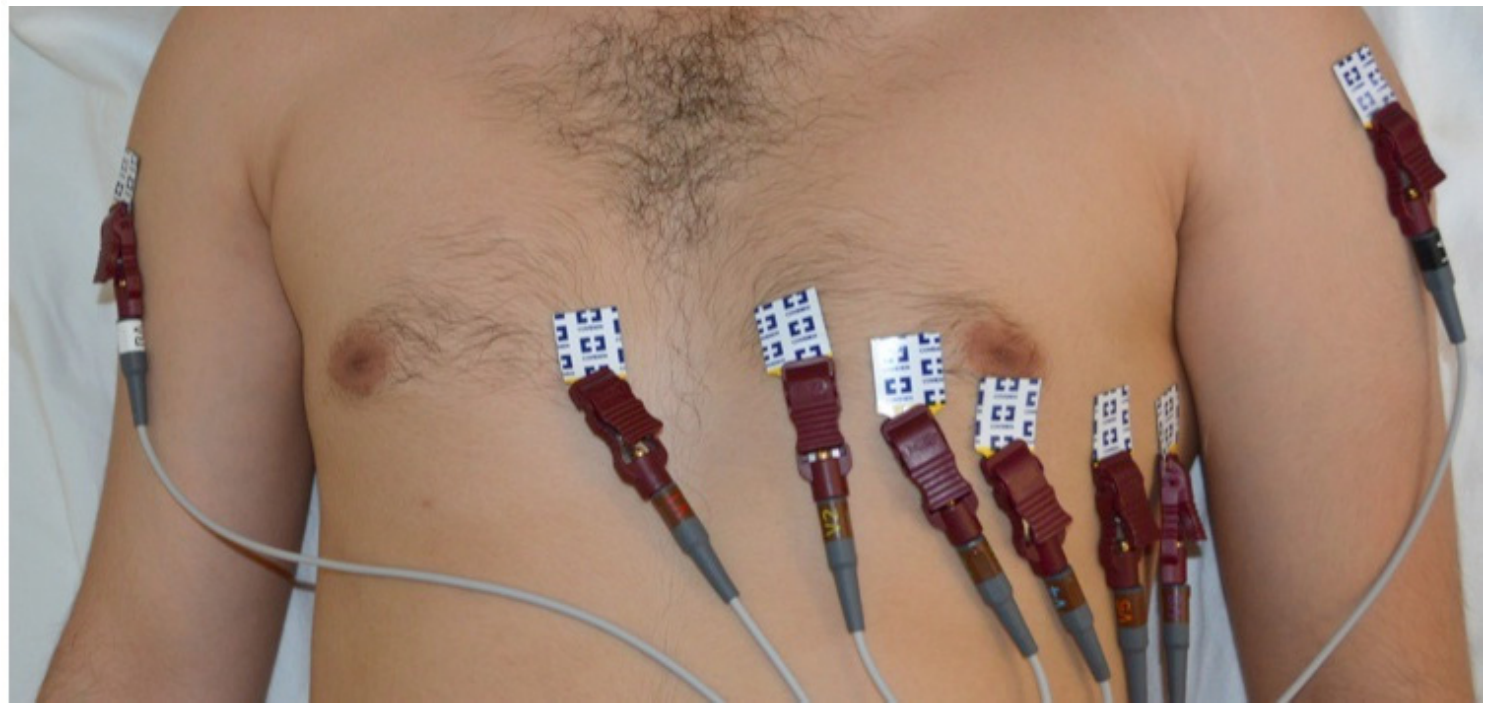

Figure 2. 12 lead ECG depicting left arm and right arm reversal. (A) ECG recording. (B) Arrows point to reversal of leads on left arm and right arm.

A

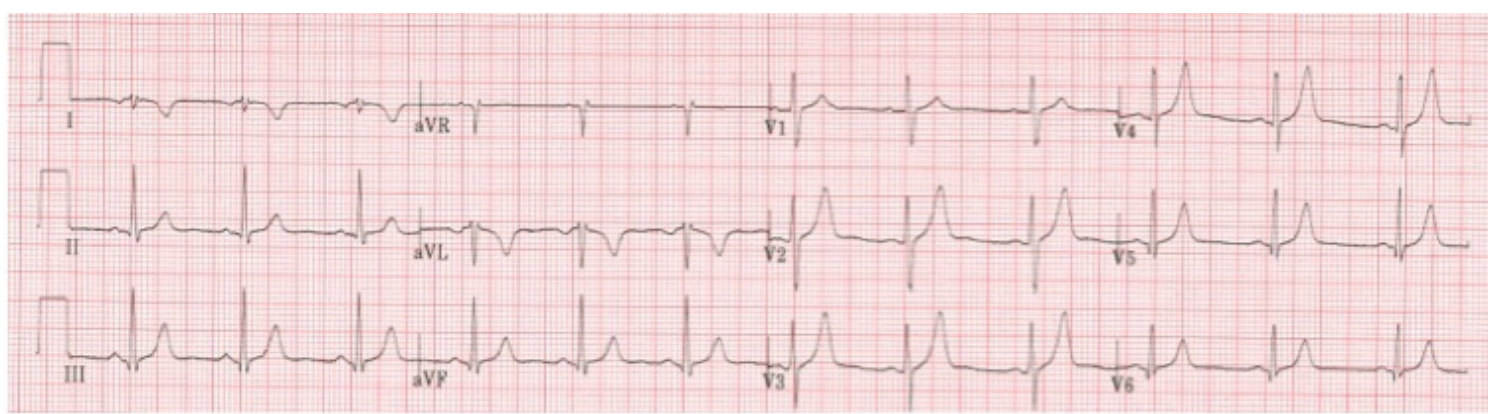

B

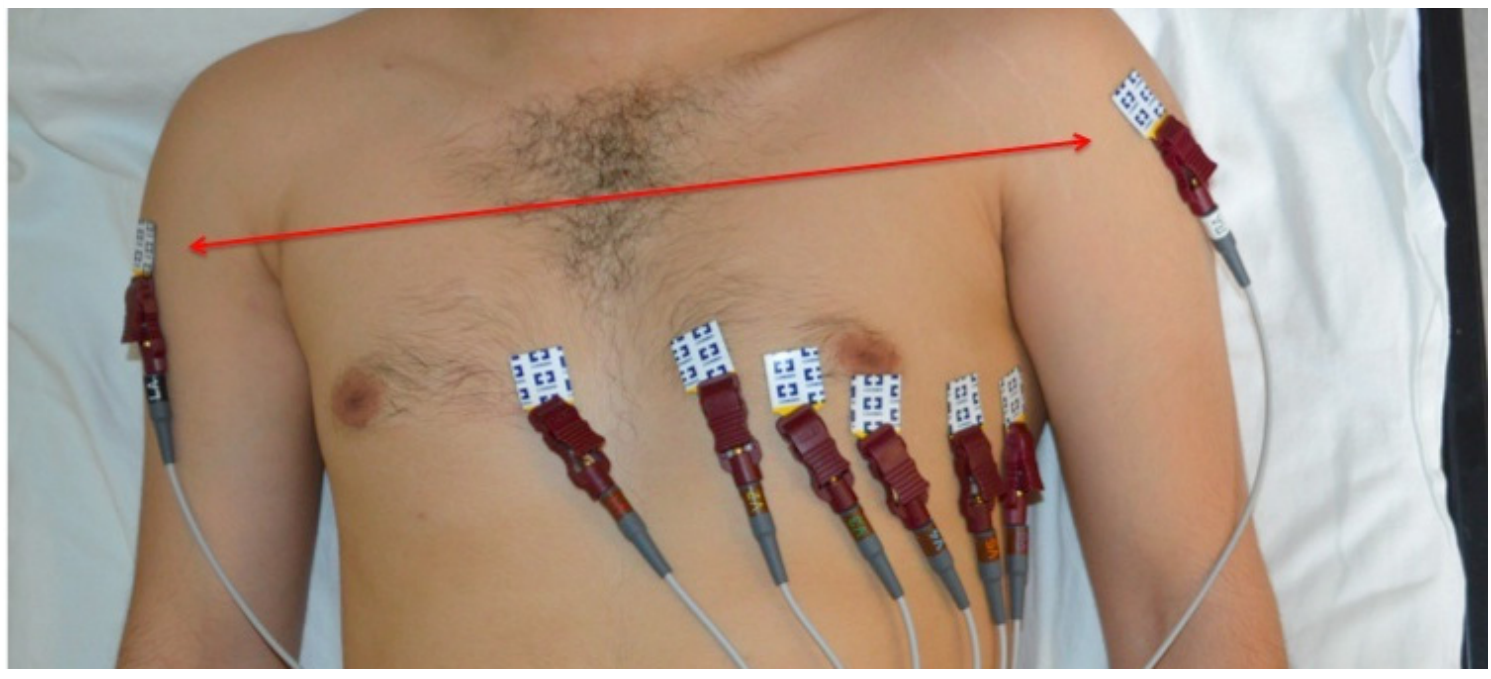




\section{Results}

\section{Left Arm and Right Arm Reversal}

Reversal of the arm electrodes is a common error. ${ }^{15}$ This produces a reversed $P$ wave, QRS complex, and T wave in leads I and aVL (Figure 2). These findings can mimic an ectopic atrial rhythm, however, the inverted $\mathrm{P}$ waves should not be followed by QRS inversion in ectopic atrial rhythms. In the case presented here, the negative polarity of the QRS and T wave ruled out ectopic atrial rhythm. ${ }^{16}$ Inversion of P waves and QRS complexes in leads I and aVL can also be misinterpreted as dextrocardia. Dextrocardia can be ruled out as a diagnosis by noting normal $\mathrm{R}$ wave progression in the precordial leads in the con text of an arm reversal. Dextrocardia will typically present with poor $\mathrm{R}$ wave progression in the precordial leads, as ventricular depolarization occurs in the opposite direction. ${ }^{7,15}$

\section{Left Leg and Right Leg Reversal}

Reversal of the leg leads does not produce a significantly altered ECC, as seen in Figure 3. It has therefore been hypothesized that this error goes unrecognized in many cases. ${ }^{15,16}$

\section{Left Arm and Left Leg Reversal}

Reversal of the left limb leads can create subtle changes that are easily missed. ${ }^{16}$ This reversal results in Einthoven's triangle rotating 180 degrees vertically around an axis formed by aVR. Therefore, a left arm and left leg reversal can be confirmed if the P wave amplitude is greater in lead I than in lead II and/ or a positive component of the terminal $P$ wave is observed in lead III (Figure 4). This finding is known as the "Abdollah sign". .17

\section{Left Arm or Right Arm and Right Leg Reversal}

If a reversal involves the right leg and one of the arms, the potential difference recorded between the legs will be zero.? This results in a "flat-line" or pseudoasystole of an isolated lead. Reversal of the left arm and right leg will result in pseduoasystole in lead III (Figure 5). ${ }^{15,16}$ Similarly, reversal of the right arm and right leg results in pseudoasystole in lead II (Figure 6).78,15

The presence of low voltages in the limb leads could suggest a diagnosis of anything that "dampens" the reading between the heart and a recording electrode, such as a pericardial effusion or amyloidosis. However, low voltages in all the leads are required for such a diagnosis. In the case presented here, while there are low amplitudes and voltages, they are isolated to one limb lead. Furthermore, the precordial leads are all normal, making a lead reversal the most likely diagnosis. ${ }^{8}$

\section{Right Arm and Left Leg Reversal}

Reversal of the right arm and left leg electrodes produces a generalized inversion of all the frontal plane leads (I, II, III, aVF) except aVL. Furthermore, lead aVR is upright. This recording can closely mimic a chronic phase inferior myocardial infarction due to inverted T waves and QS complexes in leads II, III and aVF (Figure 7). However, a high index of suspicion is required to detect lead reversal, as lead I and aVR will not be inverted from baseline in an inferior myocardial infarction. ${ }^{18}$

\section{Dual-Lead Reversal}

Reversal of right arm with right leg, and left arm with left leg can have several physiologic mimics (Figure 8). The low voltage

Figure 3. 12 lead ECG depicting left leg and right leg reversal. (A) ECG recording. (B) Arrows point to reversal of leads on left leg and right leg.

A

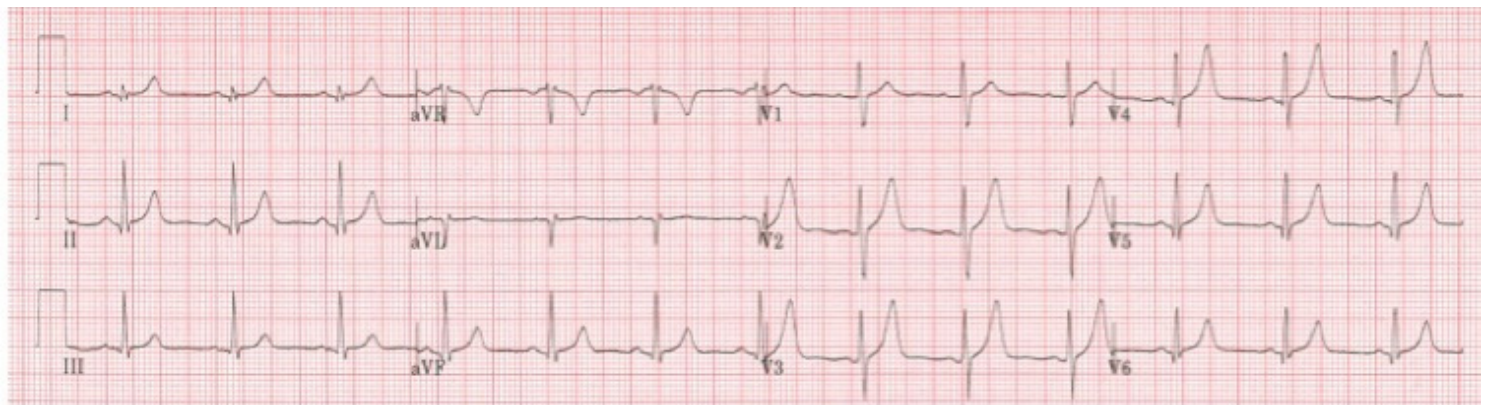

B

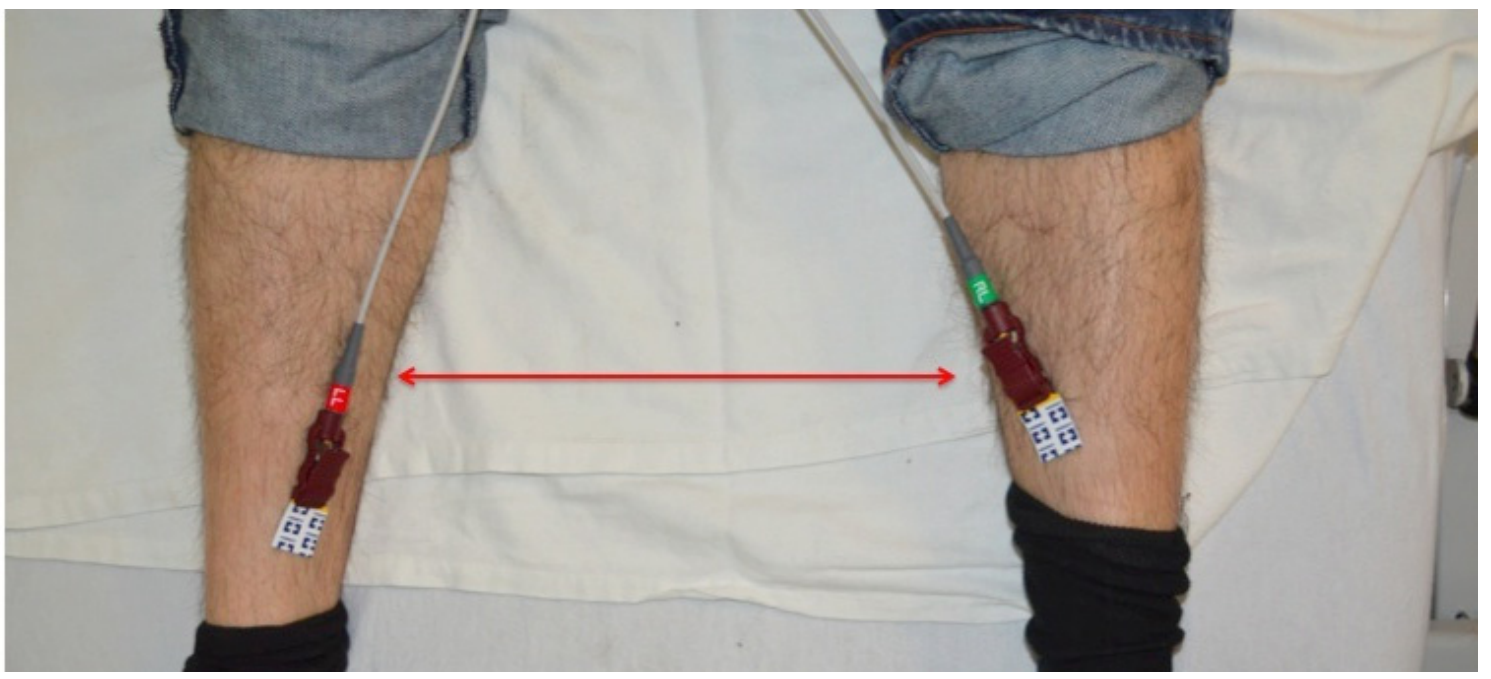


Figure 4. 12 lead ECG depicting left arm and left leg reversal. (A) ECG recording. (B) Arrows point to reversal of leads on left arm and left leg.

A

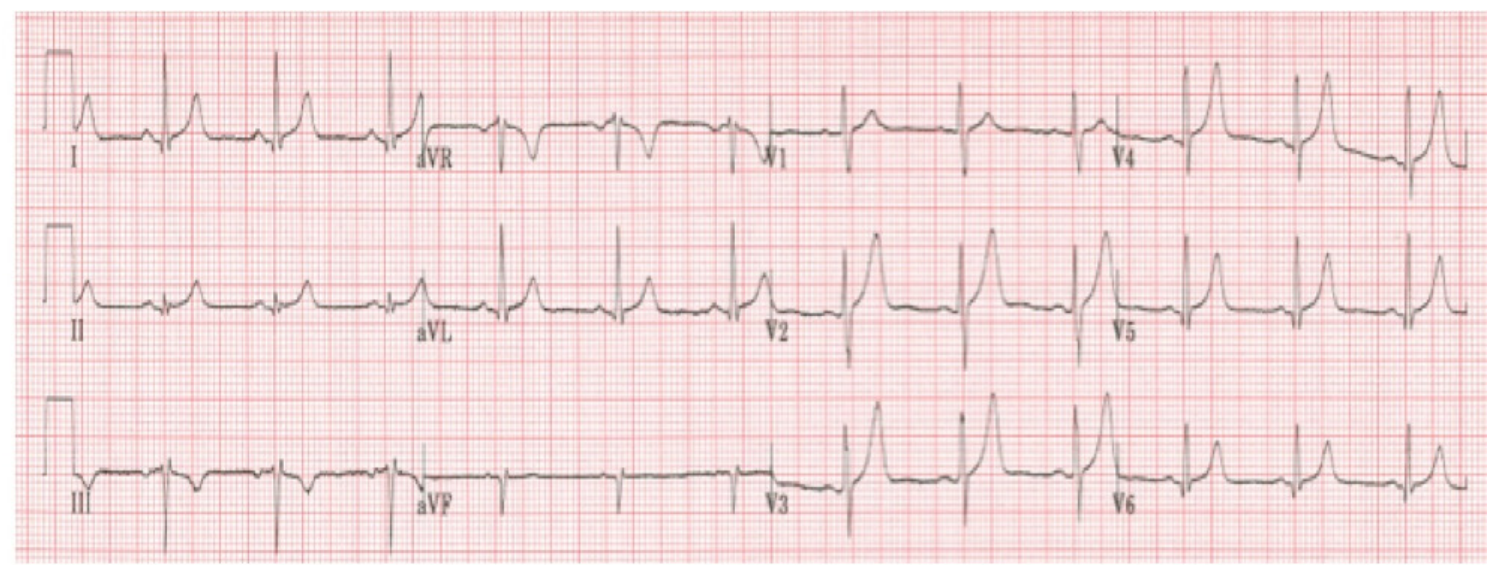

B
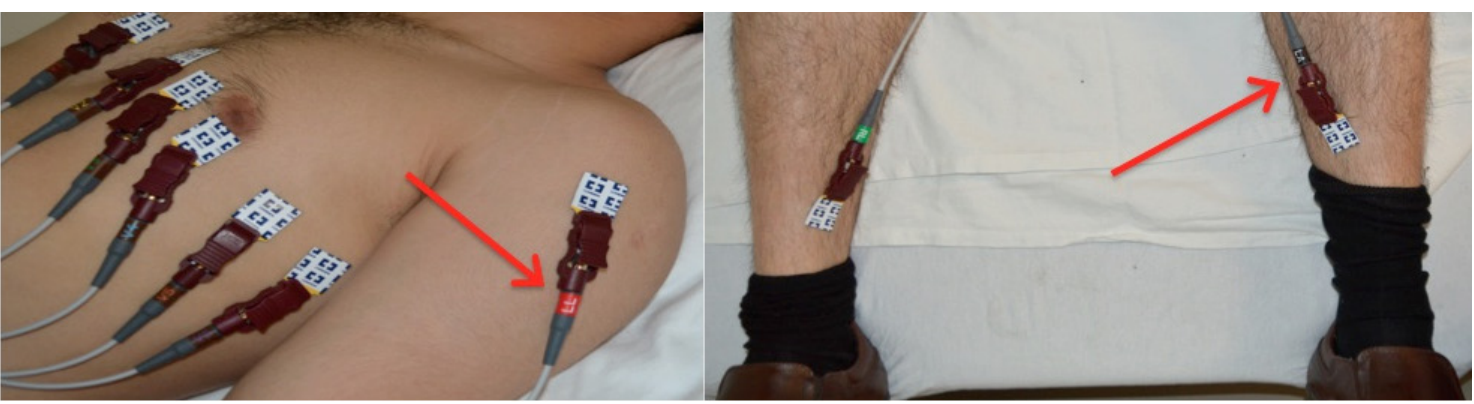

in the limb leads is suggestive of a pericardial effusion. However, the precordial leads are normal, making this diagnosis less likely. ${ }^{19}$ Low atrial rhythm might similarly be suspected due to inverted $\mathrm{P}$ waves in leads II, III and aVF. However, the diagnosis of low atrial rhythm further relies on QRS complexes being usually upright in the inferior leads along with upright $P$ waves in aVR. In this case, although $P$ wave inversions are present in the inferior leads, the other electrocardiographic findings are absent, suggesting that it is unlikely to be a low atrial rhythm. ${ }^{20}$ As discussed previously, a differential diagnosis of dextrocardia can be eliminated due to normal $R$ wave progression in the precordial leads. ${ }^{7,15}$

The ECG of a dual-lead reversal can be identified by several findings. These findings are a result of a complete reversal of the Einthoven triangle. ${ }^{8}$ Firstly, inversion of $\mathrm{P}$ waves and QRS complexes can be seen in the three inferior leads (II, III and aVF). The reversal also leads to an upright QRS complex in aVR mimicking a false left-axis deviation. Lastly, low voltages are seen in the limb leads, but not in the precordial leads. One may rule out a left arm and left leg exclusive reversal through the absence of the Abdollah sign, and a right arm and right leg exclusive reversal through the absence of pseudoasystole in lead II. 8,21

\section{Discussion}

Proper ECG recording facilitates appropriate interpretation and diagnosis. Electrode misplacements can lead to morphological changes on ECG that could potentially be interpreted as ischemic or arrhythmogenic in origin. Therefore, recognition of the patterns seen in improper lead positioning is essential to avoid incorrect diagnoses and unnecessary treatments. ${ }^{11,22,23}$
Electrode misplacements are a relatively frequent finding, present in $0.4 \%$ of ECGs done in outpatient clinics, and even more common at $4 \%$ in intensive care units. ${ }^{7}$ Several key findings on an ECG can help clinicians identify potential signs of electrode misplacements. In order to systematically identify these telltale clues, mnemonics to remember common errors and recognize their findings have been previously proposed. The REVERSE mnemonic is one such tool that outlines the most frequent abnormal findings on ECG (Table 1).? of note to our cases, three common limb lead reversals are identified in the mnemonic; (1) reversal of the left arm and right arm electrodes, (2) reversal of the right leg electrode with either of the arms, and (3) reversal of the left arm and left leg electrodes. The relevant abnormal findings on ECG have been previously explained in the results and are summarized in the table. The mnemonic also helps identify other common pitfalls in ECG recordings such as reversal of precordial leads and interference artifacts. With this mnemonic, a careful and systematic examination of ECGs will help rule out potential problems with the recording. Interestingly, although placement errors can mimic pathologies, pathologies exist that can also mimic placement errors. ${ }^{24}$ This further emphasizes the importance of careful interpretation of ECGs. Continued efforts to actively recognize lead reversals may help to avoid medical errors in diagnosis and treatment.

In conclusion, the ECG continues to be one of the most useful diagnostic tools in modern medicine. Health care providers initiate therapy based on the appropriate interpretation of ECGs. Systematic recognition of common ECG placement errors will help clinicians avoid unnecessary therapeutic interventions and enable them to obtain a properly recorded ECG. 
Figure 5. 12 lead ECG depicting left leg and right leg reversal. (A) ECG recording. (B) Arrows point to reversal of leads on left leg and right leg.

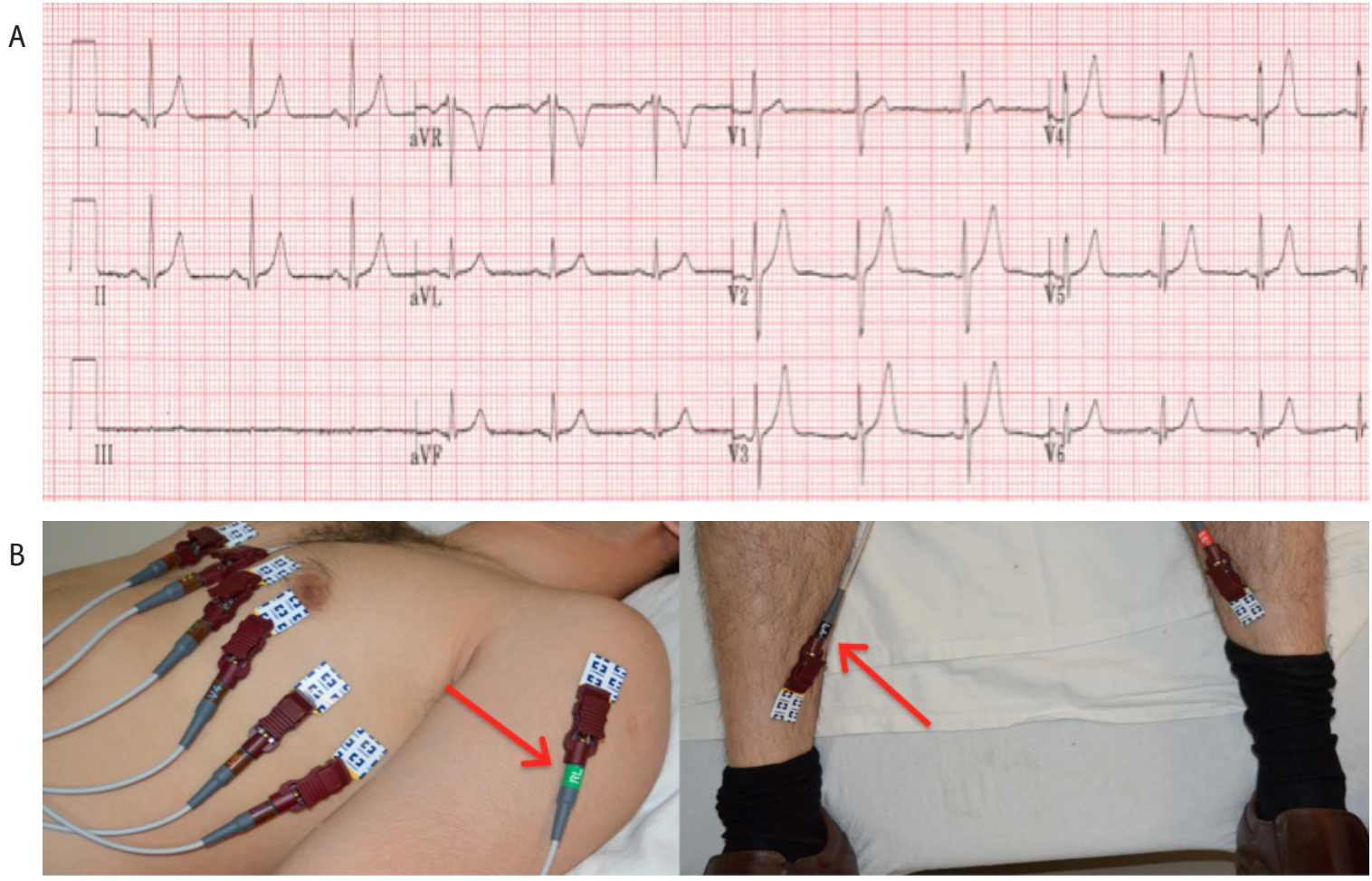

Figure 6. 12 lead ECG depicting right arm and right leg reversal. (A) ECG recording. (B) Arrows point to reversal of leads on right arm and right leg.

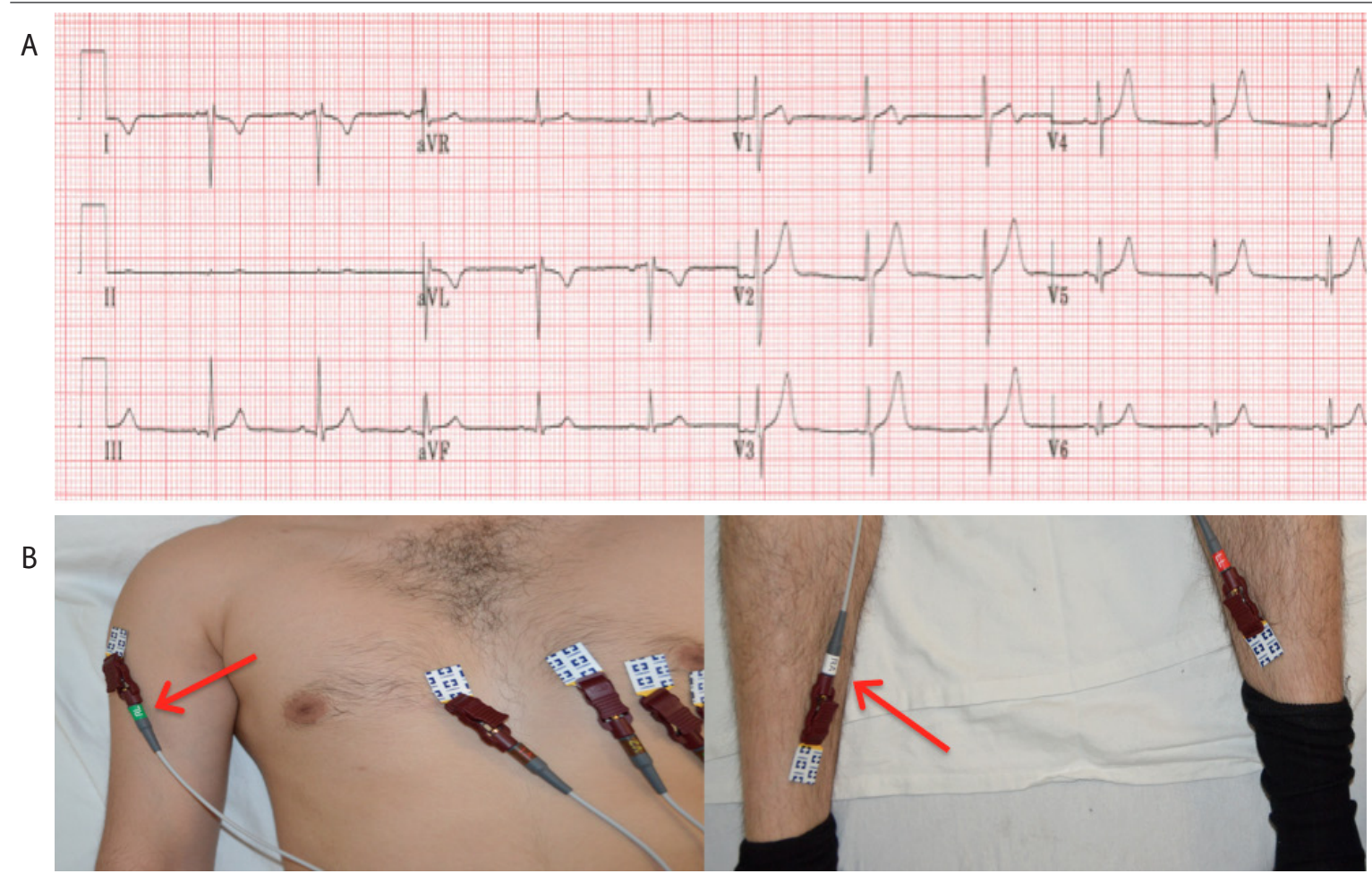


Figure 7. 12 lead ECG depicting right arm and left leg reversal. (A) ECG recording. (B) Arrows point to reversal of leads on right arm and left leg.

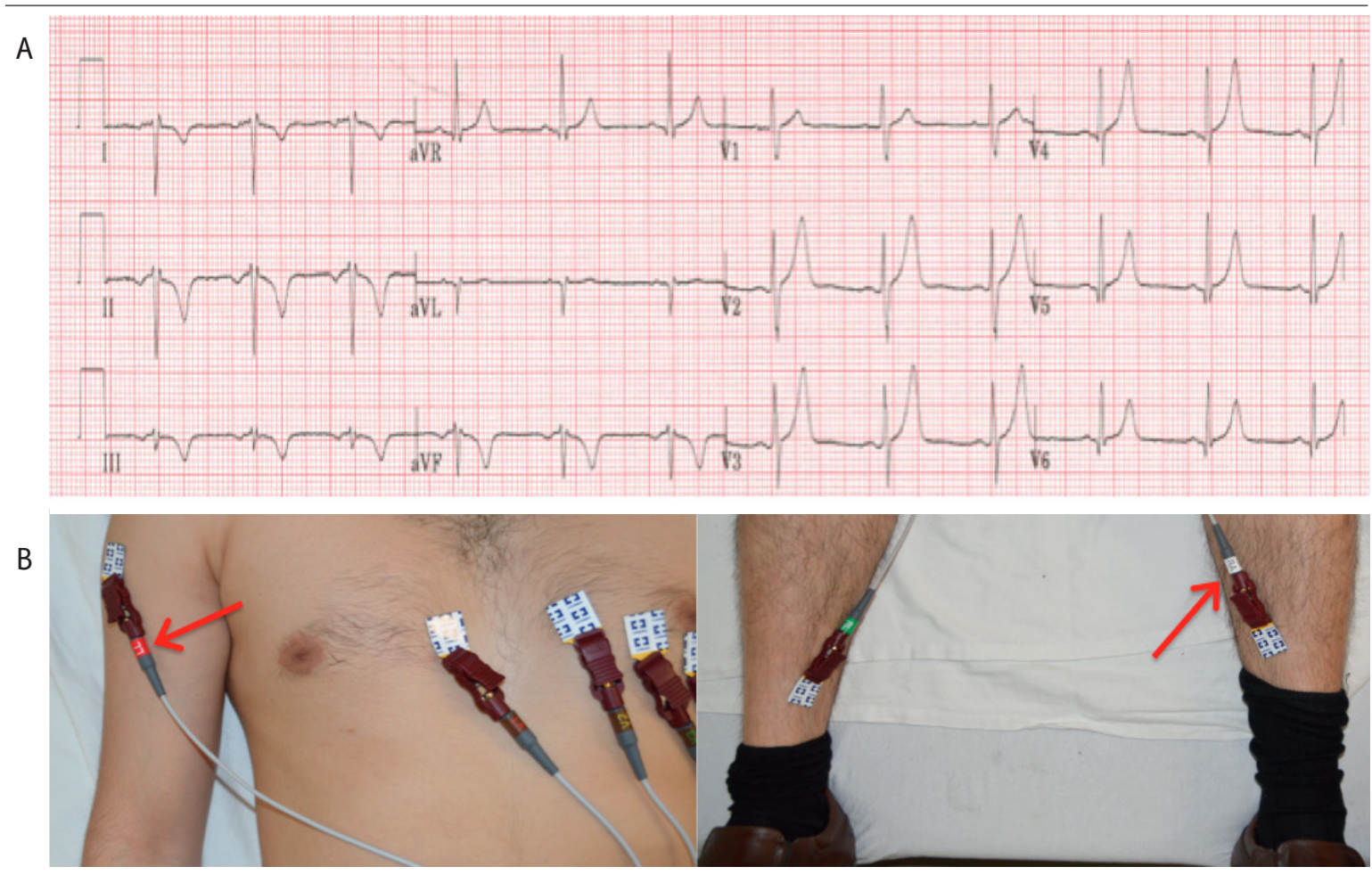

Figure 8. 12 lead ECG depicting dual-lead reversal. (A) ECG recording. (B) Arrows point to reversal of leads on right and left arms and legs.

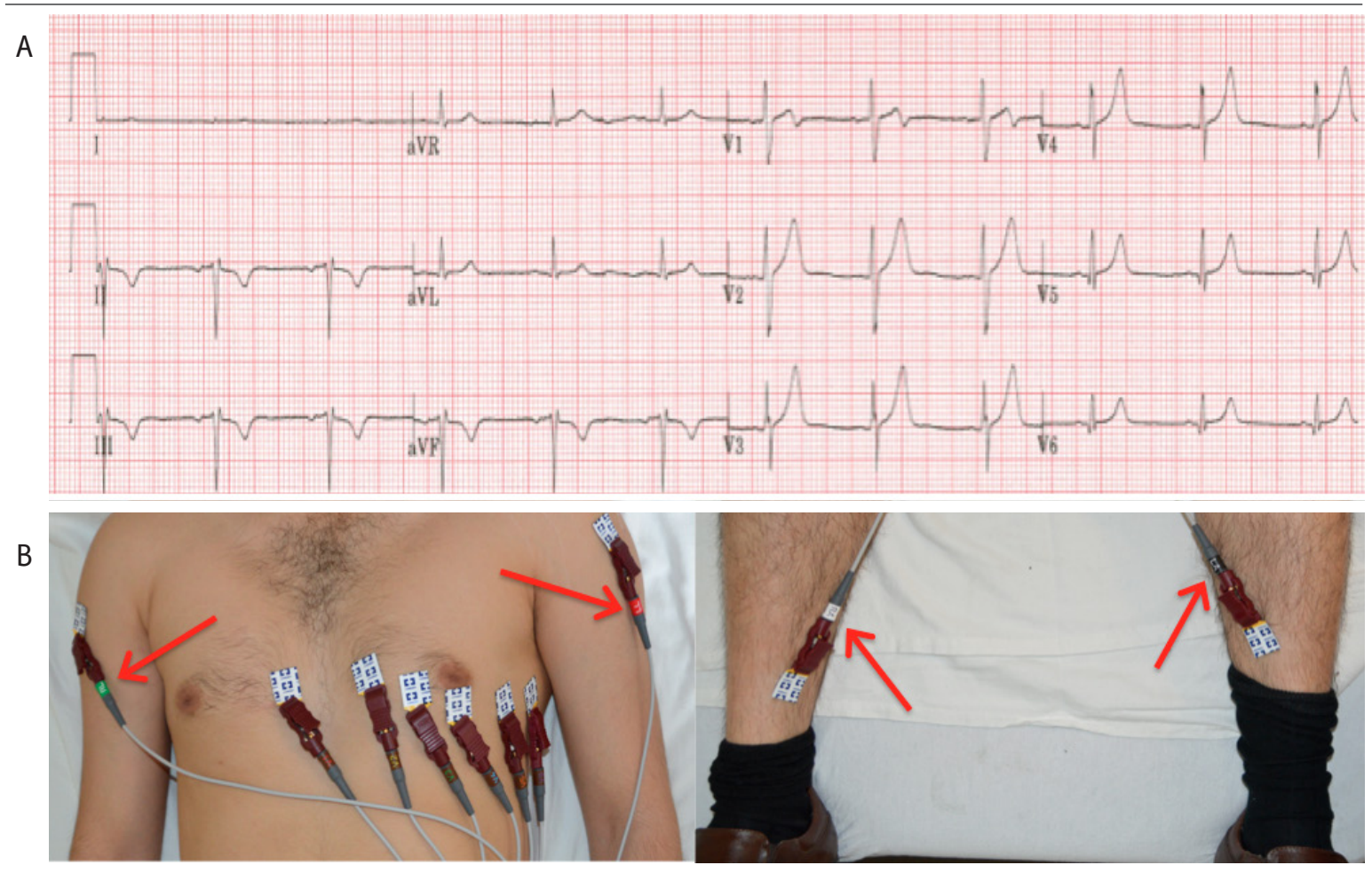




\section{References}

1. Pérez-Riera AR, Barbosa-Barros R, Baranchuk A. Current value of the electrocardiogram in the 21st century. Austin J Clin Cardiolog. 2014 Feb 24;1(2):13.

2. Mason JW, Hancock EW, Gettes LS, et al. Recommendations for the standardization and interpretation of the electrocardiogram: Part II: Electrocardiography diagnostic statement list a scientific statement from the American heart association electrocardiography and arrhythmias committee, council on clinical cardiology; the American college of cardiology foundation; and the heart rhythm society endorsed by the international society for computerized electrocardiology. J Am Coll Cardiol. 2007 Mar 13;49(10):1128-35.

3. Kligfield P. Gettes LS. Bailey JJ, et al. American Heart Association Electrocardiography and Arrhythmias Committee,Council on Clinical Cardiology. American College of Cardiology Foundation. Heart Rhythm Society. Recommendations for the standardization and interpretation of the electrocardiogram: Part I: The electrocardiogram and its technology a scientific statement from the american heart association electrocardiography and arrhythmias committee, council on clinical cardiology; the american college of cardiology foundation; and the heart rhythm society endorsed by the international society for computerized electrocardiology. J Am Coll Cardiol. 2007 Mar 13;49(10):1109-27.

4. Rajaganeshan R, Ludlam CL, Francis DP, Parasramka SV, Sutton R. Accuracy in ECG lead placement among technicians, nurses, general physicians and cardiologists. Int J Clin Pract. 2008 Jan;62(1):65-70.

5. Sejersten M, Pahlm 0, Pettersson J, et al. Comparison of EASI-derived 12-lead electrocardiograms versus paramedic-acquired 12-lead electrocardiograms using mason-likar limb lead configuration in patients with chest pain. J Electrocardiol. 2006 Jan;39(1):13-21.

6. Hoffman I. Einthoven's left foot: A plea for disciplined electrode placement. J Electrocardiol. 2008 May-Jun;41(3):205-6.

7. Baranchuk A, Shaw C, Alanazi $\mathrm{H}$, et al. Electrocardiography pitfalls and artifacts: The 10 commandments. Crit Care Nurse. 2009 Feb;29(1):67-73.

8. Koppikar S, Shaw C, Baranchunk A. ECG quiz: A tale of an abnormal ECG. J Electrocardiol. 2014 Jan-Feb;47(1):123-5.

9. Rudiger A, Hellermann JP, Mukherjee R, Follath F, Turina J. Electrocardiographic artifacts due to electrode misplacement and their frequency in different clinical settings. Am J Emerg Med. 2007 Feb;25(2):174-8.

10. Batchvarov VN, Malik M, Camm AJ. Incorrect electrode cable connection during electrocardiographic recording. Europace. 2007 Nov;9(11):1081-90. 11. Knight BP, Pelosi F, Michaud GF, Strickberger SA, Morady F. Clinical consequences of electrocardiographic artifact mimicking ventricular tachycardia. $\mathrm{N}$ Engl J Med. 19991000 Oct 21;341(17):1270-4.

12. Harrigan R, Chan T, Brady W. Diagnosis: Limb electrode reversal. Emergency Medicine News. 2004 June;26(6):18-20.

13. Garcia-Niebla J, Llontop-Garcia P, Valle-Racero JI, Serra-Autonell G, Batchvarov VN, de Luna AB. Technical mistakes during the acquisition of the electrocardiogram. Annals of Noninvasive Electrocardiology. 2009 0ct;14(4):389-403. 14. Report of committee on electrocardiography, american heart association. recommendations for standardization of leads and of specifications for instruments in electrocardiography and vectorcardiography. Circulation. 1967 Mar;35(3):583-602.

15. Drew BJ. Pitfalls and artifacts in electrocardiography. Cardiol Clin. 2006 vii; Aug;24(3):309-15.

16. Peberdy MA, Ornato JP. Recognition of electrocardiographic lead misplacements. Am J Emerg Med. 1993 Jul;11(4):403-5.

17. Abdollah H, Milliken JA. Recognition of electrocardiographic left arm/left leg lead reversal. Am J Cardiol. 1997 Nov 1;80(9):1247-9.

18. Ho KK, Ho SK. Use of the sinus $P$ wave in diagnosing electrocardiographic limb lead misplacement not involving the right leg (ground) lead. J Electrocardiol. $2001 \mathrm{Apr} ; 34(2): 161-71$.

19. Eisenberg MJ, de Romeral LM, Heidenreich PA, Schiller NB, Evans GT,Jr. The diagnosis of pericardial effusion and cardiac tamponade by 12-lead ECG. A technology assessment. Chest. 1996 Aug;110(2):318-24.

20. de Voogt WG, van Mechelen R, Scheffer M, van Miltenburg van Zijl AJ, Elhendy AA. Electrocardiographic characteristics in low atrial septum pacing. J Electrocardiol. 2005 Apr;38(2):166-70.

21. Hoffman I. A flatline lead I results from bilateral arm-to-leg electrode exchange. J Electrocardiol. 2008 Sep-0ct;41(5):388-90.

22. Srikureja W, Darbar D, Reeder CS. Tremor-induced ECG artifact mimicking ventricular tachycardia. Circulation. 2000 Sept 12;102(11):1337-8.

23. Knight BP, Pelosi F, Michaud GF, Strickberger SA, Morady F. Physician interpretation of electrocardiographic artifact that mimics ventricular tachycardia. Am J Med. 2001 Apr 1;110(5):335-8.

24. Wieters JS, Carlin JP, Morris A. Case report: An electrocardiogram of spontaneous pneumothorax mimicking arm lead reversal. J Emerg Med. 2014 May; $46(5): 620-3$.

\section{Acknowledgments}

None.

Conflict of Interest Statement A Funding

The Authors have no funding, financial relationships or conflicts of interest to disclose.

\section{Author Contributions}

Conception and design the work/idea: AR AB. Collect data/obtaining results: AR SK CS. Analysis and interpretation of data: AR SK CS AB. Write the manuscript: AR SK. Critical revision of the manuscript: AR SK AB. Approval of the final version: AB. Contribution of patients or study material: SK CS AB. Administrative or technical advice: CS.

Cite as:

Rosen AV, Koppikar S, Shaw C, Baranchuk A. Common ECG Lead Placement Errors. Part I: Limb Lead Reversals. Int J Med Students. 2014 Jul-0ct;2(3):92-8. 